\title{
Role of CT and MRI in Assessment of Temporal Bone Pre and Post Cochlear Implantation
}

Tarek Mohamed El-Zayat, Mohamed Salah Elfeshawy, Ahmed Hassan Khashaba, Mohamed Ezzat Abd El-Raouf

Department of Radiology, Faculty of Medicine, Al-Azhar University.

*Corresponding author: Mohamed Ezzat Abd El-Raouf, Mobile: (+20)01113066065, E-Mail: ezzatmohamed15@gmail.com

\begin{abstract}
Background: MDCT and MRI plays a critical role in evaluation and management of different causes of hearing loss which require many therapeutic techniques including cochlear implantation. MDCT has proven its efficacy in the postoperative imaging of cochlear implant patients. CT confirms the intracochlear position of the implant. It has also been shown that malpositioning and kinking can be detected by CT imaging.

Aim of the Work: To evaluate the role of various imaging modalities (CT and MRI) in pre and postoperative evaluation of cochlear implant candidates.

Patients and Methods: The study included a total of 20 patients referred to the Radiodiagnosis Department from the E.N.T Department in EL-Galaa Military Hospital. The patients had bilateral severe to profound sensorineural hearing loss (SNHL). CT and MRI were done for assessment of the cochlear state prior to the cochlear implantation operation. Postoperative CT was done to underline the position of implanted electrode.

Results: The study was performed on 8 males (40\%) and 12 females (60\%). Only 17 patients (85\%) underwent cochlear implantation, the other 3 cases $(15 \%)$ were diagnosed as Michel deformity, Cochlear hypoplasia and Labyrinthine ossificans. Full electrode array insertion was reported in all cases underwent of cochlear implantation.

Conclusion: Preoperative CT and MRI assessment is critical for determining implant candidacy. Postoperative CT confirms the intracochlear position of the implant.

Keywords: CT, MRI, temporal bone, petrous, cochlear implantation, preoperative, postoperative.
\end{abstract}

\section{INTRODUCTION}

The cochlea implant is a high-tech device that is inserted into the cochlea of patients with acute and severe bronchial hearing loss who have not benefited from conventional hearing amplification devices ${ }^{(\mathbf{1})}$.

Candidates for the cochlear implant undergo preoperative assessment involving clinical, speech therapeutic, psychological, social criteria and imaging of the cochlear region to identify the etiology of hearing loss, findings that may contraindicate surgery and helping to select the ear to be implanted ${ }^{(2)}$. Cochlear implants aim to provide complex sound analysis by stimulating auditory cortex over a wide range of frequencies. To achieve this goal, the implant must be placed well within the cochlear lumen.

Therefore a detailed preoperative and postoperative radiological assessment of the temporal bone has become vital for cochlear implantation ${ }^{(3)}$. MDCT and MRI plays a critical role in evaluation and management of different causes of hearing loss which require many therapeutic techniques including cochlear implantation (4). Multi-slice CT has proven its efficacy in the postoperative imaging of cochlear implant patients. CT confirms the intracochlear position of the implant. It has also been shown that malpositioning and kinking can be detected by $\mathrm{CT}$ imaging ${ }^{(5)}$.

\section{PATIENT AND METHODS}

This study included 20 patients (8 males and 12 females) with severe to profound bilateral sensorineural hearing loss (SNHL). All patients were referred from ENT Department of El Galaa Military Hospital. CT and MRI were performed as a part of preoperative assessment in Radiodiagnosis Department.

The study was approved by the Ethics Board of Al-Azhar University and an informed written consent was taken from each participant in the study.

CT was done after cochlear implantation to ensure intracochlear position of inserted electrode. An approval of the study was obtained from El Galaa Military Hospital academic and ethical committee. Written consent was taken from patients to participate in this study. Imaging for the pediatric population was performed under sedation or short acting general anesthesia. Low-dose pediatric HRCT protocols are used to keep radiation doses to a minimum.

\section{Patient selection (Inclusion criteria):}

Cochlear implants are intended for patients with bilateral severe to profound, pre or post-linguistic SNHL and who demonstrate limited benefit from amplification (6). The goal of clinical and imaging evaluation is to select those patients who will benefit the most from implantation ${ }^{(7)}$. The decision to operate is made after a thorough evaluation by a multidisciplinary team ${ }^{(8)}$. 
Role of CT and MRI in Assessment of Temporal Bone...

Exclusion Criteria: Active middle ear disease, congenital aural dysplasia and patients medically unfit for undergoing cochlear implantation ${ }^{(6)}$.

\section{Patient preparation:}

- Detailed history was taken from the parents/patient.

- Preoperative assessment involving clinical, speech therapeutic, psychological, social criteria and imaging (CT and MRI) of the cochlear region.

- Detailed explanation of the procedure to the parents/patient.

- Obtaining informed consent from the parents/patient.

Technique of CT: Imaging for the pediatric population was performed under sedation or short acting general anesthesia.

All patients were examined by MDCT laid supine with head first then axial images were obtained from the top of the petrous apex to the inferior tip of the mastoid bone with the patient's neck semi flexed. The images were transferred to workstation where MPR images were conducted for image analysis.

\section{The following parameters were used:}

The images were obtained in $1 \mathrm{~mm}$ slice thickness, 0.5 second rotation time, $0.5-1$ pitch factor, $140 \mathrm{KV}$ tube voltage, $160 \mathrm{~mA}$ tube current, a $200 \mathrm{~mm}$ scan field of view (FOV), $725 \times 725$ matrix, window level $=600$ and window width $=4000$. The image was reconstructed with

slice thickness $=0.6 \mathrm{~mm}{ }^{(9)}$.

Technique of MRI: The ideal MRI scan would be short in duration and non-strenuous for the patient and technician. It would provide images that demonstrate high spatial resolution, minimal noise, and consistent signal intensities throughout the scan ${ }^{(\mathbf{1 0})}$. Imaging for the pediatric population was performed under sedation or short acting general anesthesia. A standard head coil is used for neuro-otologic MRI examinations. Superficial coils that display the temporal bone in detail can also be used, but in order to also include the brain stem and brain it is necessary to switch to a standard head coil. Multichannel coils that enable parallel imaging are used for this purpose ${ }^{(\mathbf{1 0})}$.

\section{MRI was performed with following sequences:}

Axial T2-weighted obtained through the whole brain using the following parameters (TR: $4000 \mathrm{~ms}$; TE: $100 \mathrm{~ms}$; slice thickness: $3 \mathrm{mms}$; FOV: $200 \times 200 \mathrm{mms}$; matrix: $256 \times 256$ ). Axial and coronal T1-weighted images are obtained using the following parameters :(TR: $400 \mathrm{~ms}$; TE : $9 \mathrm{~ms}$; slice thickness :2 mm; matrix : $512 \times 512 ; \mathrm{FOV}=160 \times 160 \mathrm{mms})$ Axial, coronal and reformatted sagittal oblique T2-weighted images are obtained using the following parameters: (TR: $4000 \mathrm{~ms}$;
TE: $100 \mathrm{~ms}$; slice thickness: 2 mms; FOV: 160× 160mms; matrix: 512×512). 3D FIESTA (Fast Imaging Employing Steady-state Acquisition) obtained by using the following parameters :(TR: $8 \mathrm{~ms}$; TE: $4 \mathrm{~ms}$; slice thickness: $1 \mathrm{~mm}$; matrix: $512 \times 512$; FOV $=160$ $\mathrm{x} 160 \mathrm{mms})^{(\mathbf{1 0})}$.

CT and MRI analysis: Each case was assessed for: Different abnormalities requiring cochlear implantation. Congenital vs acquired lesions. Whether cochlear implantation would be useful for the patient or not.

Postoperative MDCT: Generation of 2D reformations and $3 \mathrm{D}$ reconstruction to visualize the electrode array within the cochlea or not ${ }^{(11)}$.

Postoperative MRI: Cochlear implants are a contraindication for MRI because they are electronically activated and have a magnet. However, recent works tended to demonstrate that MRI exam did not alter function of certain cochlear implant or displace a wellpositioned cochlear implant ${ }^{(\mathbf{1 2})}$.

However, this contraindication persists due to the danger of heating or of exciting currents with adverse nerve-effects. In any case, susceptibility artefacts induced by the implant are so important that it completely blurs the posterior fossa. Therefore, MR cannot be used in case of failure of the implanted device. This should encourage manufacturers to develop cochlear implant devices with an external part that can be easily removed and with an electrode-array without ferromagnetic components (i.e. tantalum, titanium, platinum, etc.) and performed with a nonmagnetic-effect shape ${ }^{(\mathbf{1 2})}$.

\section{Statistical analysis}

Recorded data were analyzed using the statistical package for social sciences, version 20.0 (SPSS Inc., Chicago, Illinois, USA). Quantitative data were expressed as mean \pm standard deviation (SD). Qualitative data were expressed as frequency and percentage.

\section{The following tests were done:}

- Independent-samples t-test of significance was used when comparing between two means.

- Chi-square $\left(\mathrm{x}^{2}\right)$ test of significance was used in order to compare proportions between two qualitative parameters.

- The confidence interval was set to $95 \%$ and the margin of error accepted was set to 5\%. The p-value was considered significant as the following:

- Probability (P-value)

- P-value <0.05 was considered significant.

- P-value <0.001 was considered as highly significant.

- P-value >0.05 was considered insignificant.

\section{RESULTS}

This study included 20 patients with bilateral severe 
to profound sensorineural hearing loss (SNHL). The study was performed on 8 males (40\%) and 12 females $(60 \%)$. The age of the total sample ranged from one year to 45 years, mean age 8.05 years.

Table (1): Distribution of patients according to age and sex group

\begin{tabular}{|c|c|c|c||}
\hline Age group jo. of Patient: & Males & Females \\
\hline 1 - 3 years & $13(65 \%)$ & $6(46.2 \%)$ & $7(53.8 \%)$ \\
\hline 4-8 years & $4(20 \%)$ & $1(25 \%)$ & $3(75 \%)$ \\
\hline > 8 years & $3(15 \%)$ & $1(33.3 \%)$ & $2(66.7 \%)$ \\
\hline Total & $20(100 \%)$ & $8(40 \%)$ & $12(60 \%)$ \\
\hline
\end{tabular}

Table (1) revealed that, in our study: The most common age group is from 1-3 years representing $65 \%$ of cases. Females were more affected than males representing $60 \%$ of the cases.

Patients were divided according to the onset of hearing loss into two main categories (Table2): prelingual (deafness before patients begins to speak), and post-lingual (deafness after acquisition of speech).

Table (2): Distribution of patients according to onset of hearing loss

\begin{tabular}{|c|c|c|}
\hline Onset & No. of patients & \% \\
\hline Pre-lingual & 12 & $60 \%$ \\
\hline Post-lingual & 8 & $40 \%$ \\
\hline
\end{tabular}

Table (2) showed that: The most common onset is the prelingual representing (60\%) of cases.

The patients were classified according to etiological factors of SNHL into two groups: congenital and acquired causes (Table 3).

Table (3): Frequency of etiological factors of SNHL among the study group.

\begin{tabular}{|c|c|c|}
\hline Aetiology & Total of SNHL & Frequency \\
\hline Congenital & 12 & $60 \%$ \\
\hline Acquired & 8 & $40 \%$ \\
\hline Total & 20 & $100 \%$ \\
\hline
\end{tabular}

Table (3) revealed that: The most common etiological factor for SNHL was congenital (found in 12 patients representing $60 \%$ of the total number of the patients).

Preoperative MDCT and MRI assessment: Most causes of hearing impairment including the external auditory canal, middle ear space and the cochlea are best visualized with computed tomography scan and magnetic resonance imaging of the temporal bone (Table 4).
Table (4): MDCT and MRI findings of the inner ear of the 20 patients

\begin{tabular}{|l|c||c||}
\hline MDCT findings & $\begin{array}{c}\text { No. of } \\
\text { patients }\end{array}$ & \\
\hline Congenital & 5 & \\
Mondini deformity & 2 & \\
Vestibular aqueduct syndrome & 1 & $25 \%$ \\
Michel deformity & 1 & \\
Cochlear hypoplasia & 1 & \\
\hline Post meningitic calcification & 1 & $5 \%$ \\
\hline Normal & 14 & $70 \%$ \\
\hline Total & 20 & 100 \\
\hline
\end{tabular}

Table (4) revealed that: The majority of the patients had normal study of the inner ear (14 out of 20 patients). Congenital malformation of inner ear was detected in only 5 cases representing $25 \%$ of cases.

In our study:

Only 17 patients (85\%) underwent cochlear implantation (Table 5), the other three cases were diagnosed as:

- Michel deformity: This case has been excluded from undergoing cochlear implantation because of absence of vestibulocochlear structures bilaterally.

- Cochlear hypoplasia: This case has been excluded from undergoing cochlear implantation because it has bilateral cochlear hypoplasia (only one turn or a partial turn is seen) and bilateral hypoplastic cochlear nerves.

- Labyrinthine ossificans: This case has been excluded from undergoing cochlear implantation because of bilateral completely ossified of both cochlea which affects insertion of the wire during cochlear implantation operation.

Table (5) Frequency of cochlear implantation in the patients

\begin{tabular}{|l|c|c||}
\hline Cochlear implantation & No. of patients & $\%$ \\
\hline $\begin{array}{l}\text { Underwent cochlear } \\
\text { implantation }\end{array}$ & 17 & $85 \%$ \\
\hline $\begin{array}{l}\text { Not "underwent } \\
\text { cochlear implantation" }\end{array}$ & 3 & $15 \%$ \\
\hline Total & 20 & $100 \%$ \\
\hline
\end{tabular}

Table (5) revealed that 17 patients $(85 \%)$ underwent cochlear implantation.

Postoperative MDCT assessment: MDCT is necessary to underline the position of implanted electrode, ensure intracochlear position and detect electrode kinking and may serve as a reference. 
Role of CT and MRI in Assessment of Temporal Bone...

Full electrode array insertion was reported in all cases underwent cochlear implantation.

\section{DISCUSSION}

Cochlear implantation is the standard procedure for managing severe to profound SNHL ${ }^{(13)}$. Cochlear implants recommended for children as young as 12 months and no upper age limit ${ }^{(8)}$. Multiple models of cochlear implant devices present. All are multichannel intracochlear array devices ${ }^{(14)}$.

The aim of this study was to to evaluate the role of various imaging modalities (CT and MRI) in pre and postoperative evaluation of cochlear implant candidates.

All cases (20 cases) underwent preoperative MDCT and MRI of both temporal bones.

Only (17 cases) underwent postoperative MDCT.

In our study, we agree with Lane et al. ${ }^{(15)}$, who found that the use of the oblique sagittal reconstructions with different angles had solved the problem with volume averaging effect at SCC imaging and diagnosis of dilated vestibular aqueduct.

Moreover, oblique sagittal reconstruction can depict the entire length of the tympanic and mastoid segments of the facial nerve.

In our study, we agree with Chavhan et al. ${ }^{(16)}$, that CISS/FIESTA-C has become a sequence of choice for evaluating the cranial nerves. It shows dark cranial nerves against a background of bright CSF. Cerebellopontine angle cistern lesions and cranial nerves VII and VIII in the internal auditory canal and labyrinth are best evaluated with CISS/FIESTA-C.

In our study, most children with congenital SNHL showed normal inner ear morphology with congenital inner ear anomalies reported in $(41.6 \%)$. This finding agrees with Haung et al. ${ }^{(17)}$, who explained that the hearing loss is frequently due to microscopic level, which doesn't affect the appearance of the bony otic capsule or membranous inner ear. In our study, we agree with Morzaria et al. ${ }^{(18)}$ who reported that meningitis is the most common postnatal cause of acquired bilateral SNHL, as the 8 patients presented to us with post-lingual hearing loss, all of them were post meningitic.

In our study, the most common cause of the SNHL was congenital causes (representing 60\% of cases), then acquired causes (representing $40 \%$ of cases). This slightly differs from McClay et al. ${ }^{(19)}$ who reported that the SNHL described in children was due to genetic cause in $50 \%$ of their sample while, acquired and unknown causes represented $50 \%$. In our study we agree with Mackeith et al. ${ }^{(20)}$ saw that combined MDCT and MRI is better as MRI can assess the cochlear nerve anomalies like nerve absence and early stages of post meningitic labyrinthine fibrosis.

In the current study, out of the 12 patients with congenital SNHL only 5 patients (41.6\%) showed congenital malformation of their inner ears ranged from IP II, dilated vestibular aqueducts, Michel deformity and cochlear hypoplasia. This result differs from that of Gupta et al. (21) who reported that congenital malformations of the inner ear are rare anomalies; so they can be identified on imaging in about $20 \%$ of patients with congenital SNHL.

In our study we agree with Broomfield et al. ${ }^{(12)}$ that certain abnormalities of inner ear are better depicted on CT, while others are better seen on MRI. Hence, neither MDCT nor MRI of the brain and temporal bones appears to be adequate as a single imaging modality but they are complementary to each other for preoperative imaging of cochlear implantation.

In our study we agree with Arweiler et al. ${ }^{(22)}$ that MDCT has proven its efficacy in the postoperative imaging of cochlear implant patients. CT confirms the intracochlear position of the implant. It has also been shown that malpositioning and kinking can be detected by CT imaging. In our study full electrode array insertion was reported in all cases underwent cochlear implantation that's agree with Ying $\boldsymbol{e t}$ al. ${ }^{\text {(23) }}$ who reported that misplacement of the electrode occurs rarely.

\section{CONCLUSION}

Preoperative CT and MRI assessment of children with severe or profound sensorineural hearing loss (SNHL) is critical for determining implant candidacy. Both have their proponents. MDCT demonstrates the bony architecture of the temporal bone, while MRI is helpful for identifying membranous labyrinth and soft tissue abnormalities. MDCT has proven its efficacy in the postoperative imaging of cochlear implant patients. CT confirms the intracochlear position of the implant. It has also been shown that malpositioning and kinking can be detected by CT imaging.

\section{REFERENCES}

1- Barbosa MH, Felix F and Ribeiro MG (2014): Profile of patients assessed for cochlear implants. Braz J Otorhino-laryngol., 80(4): 305-10.

2-Vlastarakos PV, Nikolopoulos TP and Pappas S (2010): Cochlear implantation update: contemporary preoperative imaging and future prospects - the dual modality approach as a standard of care. Expert Rev Med Devices, 7(4): 555-67.

3- Chaturvedi A, Mohan C, Mahajan SB et al. (2006): Imaging of cochlear implants. Journal of Radiology and Imaging, 16 (3): 85392.

4- Michele B and Barry E (2008): Imaging of Hearing Loss. Otolaryngologic Clinics of North America, 41: 157-178.

5- Verbist BM, Joemai RM and Teeuwisse WM (2008): Evaluation of 4 multisection CT systems in postoperative imaging of a cochlear implant: a human cadaver and phantom study. AJNR Am J Neuroradiol., 29: 1382-1388.

6- Gifford RH (2011): Who is cochlear implant candidate? Hearing Journal, 64(16):1822. 
7-Witte RJ, Lan JI, Driscoll CL et al. (2003): Pediatric and adult cochlear implantation. Radiographics,23(5):1185-200.

8-Hodges AV and Balkany TJ (2002): Cochlear implants for sensorineural hearing loss. Hospital Physician,38(10): 22-32.

9- Joshi VM, Navlekar SK and Kishore GR (2012): CT and MR imaging of the inner ear and brain in children with congenital sensorineural hearing loss. Radiographies, 32(3): 683-698.

10- Redleaf MI, Andrews JD and Pinto JM (2009): MR Imaging, Temporal Bone. https://hospital.uillinois.edu/find-adoctor/miriam-redleaf

11- Zaghloul RAH, El-Sheikh ML, El-Baky FAA et al. (2011): Role of high resolution CT and radiography in postoperative evaluation of cochlear implantation. The Egyptian Journal of Radiol and Nuclear Medicine, 42(2): 177-184.

12- Broomfield SJ, Da Cruz M and Gibson WP (2013): Cochlear implants and magnetic resonance scans: A case report and review. Cochlear Implants Int., 14: 51-55.

13- Kang DH, Lee MJ and Lee KY (2016): Prediction of Cochlear Implant Outcomes in Patients with Prelingual Deafness. Clinical and Experimental Otorhino-laryngol, 9(3): 220-225.

14-Young JY, Ryan ME and Young NM (2014): Preoperative imaging of sensorineural hearing loss in pediatric candidates for cochlear implantation. Radio Graphies, 34(5): E133-E149.

15-Lane JI, Lindell EP, Witte RJ et al. (2006): Middle and inner ear: improved depiction with multiplanar reconstruction of volumetric CT data. Radiographics., 26(1):115-24.
16-Chavhan GB, Babyn PS, Singh M et al. (2009): MR imaging at 3.0 $\mathrm{T}$ in children: technical differences, safety issue, and initial experience. Radiographics., 29(5):1451-66.

17- Haung BY, Zadanski $C$ and Castillo $M$ (2012): Pediatric sensorineural-hearing loss, part 1: Practical Aspects for Neuroradiologists. Am J Neuroradiol., 33(2): 211-217.

18- Morzaria S, Westerberg BD and Kozak FK (2004): Systematic review of the etiology of bilateral sensorineural hearing loss in children. Int J Pediatr Otorhino-laryngol., 68:1193-98.

19- McClay JE, Booth TN and Parry DA (2008): Evaluation of pediatric sensorineural hearing loss with magnatic resonance imaging. Archives of otolaryngol- Head \& neck Surg., 146:180190.

20-Mackeith S1, Joy R and Robinson P (2012): Pre-operative imaging for cochlear implantation: magnetic resonance imaging, computed tomography, or both? Cochlear Implants Int., 13(3):133-6.

21-Gupta SS, Maheshwari SR and Kirtane MV(2009): Pictorial review of MRI/CT Scan in congenital temporal bone anomalies, in patients for cochlear implant. Indian J Radiol Imaging, 19(2): 99-106.

22- Arweiler-Harbeck D, Mönninghoff C and Greve J (2012): Imaging of Electrode Position after Cochlear Implantation with Flat Panel CT. ISRN Otolaryngol., 12: 228-235.

23- Ying YL, Lin JW and Oghalai JS (2013): Cochlear implant electrode misplacement: incidence, evaluation and management. Laryngoscope, 123: 757-766. 